\title{
Löhne und Lohnempfehlungen 2004 für Medizinische Praxisassistentinnen
}

\author{
Dr. med. Michel Marchev ${ }^{a}$ Dr. med. Jürg Kremo ${ }^{b}$, Elwina Kaufmann ${ }^{c}$, Fürsprecher Bruno Gutknecht ${ }^{d}$
}

Aufgrund der seit 1991 kantonal von den Ärztegesellschaften ausgearbeiteten Lohnempfehlungen für Medizinische Praxisassistentinnen werden keine gesamtschweizerischen Lohnempfehlungen mehr publiziert.

Bei individuellen Lohnverhandlungen sollen für Medizinische Praxisassistentinnen die nachstehend aufgeführten Rahmenbedingungen berücksichtigt werden.

\section{Rahmenbedingungen}

\section{Ausbildung}

- Diplom der Verbindung der Schweizer Ärztinnen und Ärzte (Vignette) bzw. Fähigkeitsausweis der FMH inkl. Strahlenschutzausweis bzw. Röntgenbewilligung;

- Eidg. Fähigkeitszeugnis Medizinische Praxisassistentin.

\section{Arbeitsbedingungen:}

Berechnungsgrundlagen

- 43-Stunden-Woche im Jahresdurchschnitt;

- 4 Wochen Ferien (Medizinische Praxisassistentinnen unter 20 und ab 50 Jahren: 5 Wochen).

Nennenswerte Abweichungen von diesen Bedingungen können auf den Lohn umgerechnet werden.

\section{Regionale Gegebenheiten}

Die Löhne sollen den regionalen Gegebenheiten angepasst werden.

\section{Monatslohn}

a Präsident, FMH-Delegierter für MPA-Fragen

b Vizepräsident, FMH-Delegierter für MPA-Fragen

c Präsidentin, Bund Schweizer Verbände Medizinischer PraxisAssistentinnen BSMPA (AGAM, ARAM, ATAM, BMPA)

d Zentralsekretär, Schweizerischer Verband Medizinischer Praxis Assistentinnen SVA
Am Jahresende ist der Medizinischen Praxisassistentin ein 13. Monatslohn auszurichten. Umfasst das Arbeitsverhältnis nicht das ganze Kalenderjahr, so ist er anteilsmässig zu bezahlen.

\section{Dienstalterszulagen}

\section{und Reallohnerhöhungen}

Die Höhe der Dienstalterszulage und einer allfälligen Reallohnerhöhung bilden ein Thema des jährlichen Qualifikationsgespräches.

\section{Teuerungsausgleich}

Die Parteien vereinbaren jeweils Ende Jahr, ob und in welchem Umfang der Lohn der Teuerung im Vorjahr angepasst wird (LIKP September: 0,5\%, Oktober: 0,5\%).

Wo der Teuerungsausgleich vertraglich vereinbart ist, muss dieser auch gewährt werden.

\section{Teilzeitarbeit im Monatslohn}

Bei Teilzeitarbeit beträgt der Bruttolohn (bei 43 Wochenstunden als Berechnungsgrundlage) $1 / 43$ eines vollen Monatslohnes, multipliziert mit der Anzahl der vereinbarten Wochenarbeitsstunden.

\section{Stundenlohn}

Bei sehr geringer und gleichzeitig unregelmässiger Arbeitszeit empfiehlt sich die Ausrichtung eines Stundenlohnes. Als Stundenlohnansatz empfehlen wir $6 \%$ eines vollen Monatslohnes (13. Monatslohn ist anteilsmässig darin enthalten). Zusätzlich muss auf diesem Ansatz ein Ferienanteil von $8,33 \%$ ausgerichtet werden, der den üblichen 4 Wochen Ferien entspricht und auf jeder Lohnabrechnung separat auszuweisen ist (bei 5 Wochen Ferien 10,64\%, bei 6 Wochen Ferien 13,04\% usw.). Diese Berechnung gilt auch bei der Auszahlung von Überstunden.

\section{Überstunden}

Die Überstundenregelung hat immer wieder zu Rückfragen geführt. Anlässlich der Revision des Mustervertrages Anfang 2001 ist dieser Problematik Rechnung getragen worden. In Ziff. 2 des Vertrages kann neu gewählt werden zwischen

- der Kompensation von Überstunden durch Freizeit oder Ferien gleicher Dauer, wobei der Arbeitgeber den Zeitpunkt bestimmt und

- der Entschädigung samt einem Lohnzuschlag von $25 \%$. Als Stundenlohnansatz gelten $6 \%$ eines vollen Monatslohnes zuzüglich Ferienanteil (vgl. «Stundenlohn»). 
Für alle vereinbarten Löhne gelten folgende Bestimmungen und Empfehlungen

Abzüge vom Bruttolohn

- AHV, IV, EO, ALV: 6,3 \% (AHV, IV, EO = 5,05 \%, $\mathrm{ALV}=1,25 \%$;

- Nichtberufsunfallversicherung: 1,367\% (bei einer wöchentlichen Arbeitszeit von mehr als 8 Stunden);

- Berufliche Altersvorsorge (2. Säule BVG): Arbeitnehmeranteil (normalerweise 50\%) des altersabhängigen Beitrages gemäss Versicherungsausweis.

\section{Arbeitsverträge und weitere Anstellungs- bedingungen}

Wir empfehlen nachdrücklich die schriftliche Vertragsform auf dem von der FMH und den Berufsverbänden der Medizinischen Praxisassistentinnen gemeinsam erarbeiteten Vertragsformular mit zugehörigen kantonalen Empfehlungen; Bezugsquellen:

- Verbindung der Schweizer Ärztinnen und Ärzte FMH, Elfenstrasse 18, 3000 Bern 16, Tel. 03135911 11, www.fmh.ch, E-Mail: fmhmprax@hin.ch;

- Association romande des assistantes médicales ARAM, Case postale 2034, 1002 Lausanne, tél. 02194345 64;
- Berufsverband Medizinischer Praxisassistentinnen BMPA, Sonnenbergstrasse 7, $6005 \mathrm{Lu}$ zern, Tel. 04131022 23;

- Bund Schweizer Verbände Medizinischer Praxis-Assistentinnen BSMPA, Sonnenbergstrasse 7, 6005 Luzern, Tel. 04131022 23, www.bsmpa.ch, E-Mail: bsmpa@bluewin.ch;

- Schweiz. Verband Medizinischer Praxis-Assistentinnen SVA, Postfach 6432, 3001 Bern, Tel. 03138114 43, www.sva.ch, E-Mail: sekretariat@sva.ch.

Die Berufsverbände der Medizinischen Praxisassistentinnen in Genf und im Tessin haben eine eigene, mit der jeweiligen kantonalen Ärztegesellschaft ausgearbeitete Arbeitsvertragskonvention bzw. Arbeitsverträge. Bezugsquellen:

- Association genevoise des assistantes médicales AGAM, rue des Pavillons 11, 1205 Genève, tél. 02232110 69;

- Associazione ticinese assistenti di studio medico ATAM, Via Ronchetto, 6814 Cadempino, tel. 0919947835.

Die Löhne für Lehrtöchter richten sich nach den kantonalen Empfehlungen. 